\title{
Resting Radionuclide Myocardial Perfusion Imaging in a Chest Pain Center Including an Overnight Delayed Image Acquisition Protocol
}

\author{
Michael W. Schaeffer ${ }^{1}$, Timothy D. Brennan², Judith A. Hughes ${ }^{3}$, W. Brian Gibler ${ }^{4}$, and Myron C. Gerson ${ }^{5}$ \\ ${ }^{I}$ Division of Cardiovascular Medicine, University of Cincinnati College of Medicine, Cincinnati, Ohio; ${ }^{2}$ Department of Internal \\ Medicine, Ohio State University College of Medicine, Columbus, Ohio; ${ }^{3}$ E.L. Saenger Radioisotope Laboratory, University Hospital of \\ Cincinnati, Cincinnati, Ohio; ${ }^{4}$ Department of Emergency Medicine, University of Cincinnati College of Medicine, Cincinnati, Ohio; and \\ ${ }^{5}$ Division of Cardiovascular Medicine, University of Cincinnati College of Medicine, Cincinnati, Ohio
}

\begin{abstract}
Emergency department (ED) patients with chest pain (CP) and a nondiagnostic electrocardiogram (ECG) present difficult management decisions. The purpose of this study was to investigate the utility of resting radionuclide SPECT myocardial perfusion imaging (SPECT MPI) — including an overnight delayed image acquisition protocol-in identifying patients presenting to the ED with $\mathrm{CP}$ at risk for cardiac events. Methods: Patients presenting to the ED with $\mathrm{CP}$ and a nondiagnostic ECG were prospectively enrolled and underwent chest pain center evaluation. All patients also underwent resting gated SPECT MPI using ${ }^{99 m}$ Tc-tetrofosmin tracer. Patients presenting on weeknights between $12 \mathrm{AM}$ and $6 \mathrm{AM}$ had tracer injection in the ED with image acquisition delayed until later in the morning. Patients were monitored for a 30-d occurrence of cardiac events. Results: Over a 16-mo period, 479 patients were enrolled and completed follow-up. For the prediction of 30-d cardiac events, resting SPECT MPI demonstrated a sensitivity and a specificity of $76.9 \%$ and $92.4 \%$, respectively. Positive and negative predictive values were $22.2 \%$ and $99.3 \%$, respectively. Among the 3 patients with a normal perfusion scan who suffered cardiac events, all had tracer injection several hours after resolution of CP. The overnight delayed image acquisition protocol provided a negative predictive value of $100 \%$ for the 44 patients whose image acquisition was delayed until the following morning. Conclusion: A normal resting SPECT MPI in ED patients presenting with $\mathrm{CP}$ predicts a very low occurrence of 30-d cardiac events. A delayed image acquisition protocol did not decrease the accuracy of SPECT MPI. Such a protocol may be useful in increasing the availability of this imaging modality.
\end{abstract}

J Nucl Med Technol 2007; 35:242-245

DOI: $10.2967 /$ jnmt.107.042796

\section{$\mathbf{P}$} atients often present to the emergency department (ED) with a complaint of chest pain (CP) and a nondiagnostic 12-lead electrocardiogram (ECG). Management

\footnotetext{
Received Apr. 18, 2007; revision accepted Jul. 5, 2007.

For correspondence or reprints contact: Myron C. Gerson, MD, Division of Cardiovascular Medicine, University of Cincinnati College of Medicine, 231 Albert Sabin Way, Cincinnati, Ohio 45267-0542.

E-mail: gersonml@ucmail.uc.edu

COPYRIGHT @ 2007 by the Society of Nuclear Medicine, Inc.
}

decisions with these patients are difficult. Patients with noncardiac $\mathrm{CP}$ or low-risk angina can be safely discharged from the ED with appropriate follow-up (1). Rarely, patients with true unstable angina (UA) or myocardial infarction (MI) are discharged from the ED with potentially dire consequences (2). Standard initial diagnostic evaluation lacks sufficient sensitivity for UA (2); therefore, many lowrisk patients are admitted, resulting in significant cost to the health care system. Resting myocardial perfusion imaging (MPI) using ${ }^{99 \mathrm{~m}} \mathrm{Tc}$ with gated SPECT has been shown to provide information for risk-stratification of this population beyond the standard evaluation (3-6). In this setting, radionuclide MPI has demonstrated favorable test characteristics, including a high negative predictive value for cardiac events $(3,7,8)$. Furthermore, MPI in the ED has been shown to be useful for clinical decision making (7-10) and to provide further information beyond that provided by serum biomarkers, including troponin (11). Rest MPI results also have short and intermediate prognostic implications in this setting $(12,13)$. The ability to offer this testing around the clock requires significant human resources with highly trained nuclear technologists and test interpreters. Many previous studies have excluded patients who arrived to the ED at night or on weekends because of the nonavailability of nuclear cardiology services $(9,10)$. The purpose of this study was to investigate the utility of resting gated SPECT MPI in identifying patients presenting to the ED with $\mathrm{CP}$ who are at risk for cardiac events. Our protocol, however, included radionuclide tracer injection by radiology personnel overnight with image acquisition in the morning. In addition to providing this imaging service around the clock, this protocol has the potential for substantial cost savings through decreased on-call requirements and a decreased admission rate for low-risk patients.

\section{MATERIALS AND METHODS \\ Patients}

Over a 16-mo period, patients presenting to the ED with $\mathrm{CP}$ and a nondiagnostic ECG were eligible for enrollment in the study. 
Exclusion criteria included ST-segment elevation, ST-segment depression $>1 \mathrm{~mm}$ in 2 electrically contiguous leads, history of coronary artery disease (CAD), clinical syndrome consistent with $\mathrm{UA}$, or hemodynamic instability. The $\mathrm{CP}$ center protocol consisted of ST-segment trend monitoring and serial cardiac markers (cardiac troponin $\mathrm{T}$ and creatine kinase $\mathrm{MB}$ ) at 0,3 , and $6 \mathrm{~h}$. Resting gated SPECT MPI was performed on all study patients while in the ED. In all cases, the tracer used was ${ }^{99 \mathrm{~m}} \mathrm{Tc}$-tetrofosmin, with a typical dose range of $740-1,110 \mathrm{MBq}(20-30 \mathrm{mCi})$. The protocol recommended that the radiotracer not be injected if $\mathrm{CP}$ had resolved for $>2 \mathrm{~h}$, although discretion was left to the physician. Perfusion images were obtained with an ECG-gated, dual-head SPECT camera using a low-energy, high-resolution, parallel-hole collimator. A Butterworth filter was used for reconstruction without attenuation correction, and a $20 \%$ symmetric energy window was centered on $140 \mathrm{keV}$. Interpretation of perfusion scans was performed by an attending nuclear medicine physician or cardiologist experienced in the interpretation of cardiac perfusion scans. After normal working hours, scans were interpreted remotely on a personal computer using commercially available software (Images-on-Call; Images on Call Teleradiology Networks). For the purposes of study analysis, perfusion scan results were classified as normal or abnormal. An abnormal scan was defined by an area of decreased myocardial tracer uptake or a regional area of decreased myocardial thickening. Equivocal scans were regarded as abnormal. A normal perfusion scan was defined by uniform myocardial uptake and normal regional wall thickening. Scans showing global left ventricular dysfunction or increased chamber volume in the absence of a regional perfusion defect were classified as normal with regard to ischemic findings. In addition to the usual daytime schedule for nuclear medicine technologists, an on-call technologist was available from 1:30 PM to 12 AM on weekdays. An on-call technologist provided imaging services during the daytime on weekends. Between the hours of $12 \mathrm{AM}$ and $6 \mathrm{Am}$ on weekdays, radionuclide injections took place in the $\mathrm{CP}$ center by radiology personnel trained in handling radioactive imaging material. In this subset of patients, gated SPECT image acquisition was delayed until shortly after 6 AM when nuclear medicine technologists arrived at the hospital.

Patients were prospectively enrolled and followed for $30 \mathrm{~d}$ after the ED visit. Primary endpoints included MI, abnormal coronary angiogram (defined by at least one coronary stenosis $>50 \%$ severity), percutaneous coronary intervention (PCI), coronary artery bypass grafting (CABG), ventricular tachycardia/ventricular fibrillation (VT/VF) arrest, heart failure admission, or cardiac-related death. Follow-up consisted of medical records review, phone contact, mail contact, and review of state and national death registries. The research protocol was approved by the institutional review board.

\section{Statistical Analysis}

Test characteristics were determined using a $2 \times 2$ matrix of the categoric variables of a normal or abnormal perfusion scan and the presence or absence of 30-d cardiac events as described. The Fisher exact test was applied to determine nonrandom association between the variables. The statistical significance level was set at $P<0.05$.

\section{RESULTS}

Four-hundred eighty-four patients were enrolled. Five were lost to follow-up, resulting in 479 patients in the final analysis. The study population demographics are shown in Table 1 . The age ranged from 20 to $80 \mathrm{y}$ with a mean age of approximately $45 \mathrm{y}$. The ratio of males to females was approximately equal. African-Americans made up the largest racial demographic, followed by Whites. Cardiovascular risk factor prevalence was typical for a low-risk population with the exception of smoking and cocaine abuse, both of which were high. Cardiovascular medication use was low in the study population. No individual class of medication was used in $>10 \%$ of the study population.

Subjects underwent a protocol-driven evaluation in the $\mathrm{CP}$ center and received gated SPECT MPI. Four-hundred thirty-four patients had normal resting MPI and 45 had abnormal MPI consistent with ischemia. In the normal MPI group, $3(0.7 \%)$ patients had cardiac events. In the abnormal MPI group, $10(22.2 \%)$ patients had cardiac events (Table 2). The difference in cardiac events between the 2 groups was highly significant statistically $(P<0.001)$. The test characteristics of gated SPECT MPI in predicting 30-d events were as follows: sensitivity, 76.9\%; specificity, 92.4\%; positive predictive value, $22.2 \%$; and negative predictive value, $99.3 \%$.

Among the 3 patients in the normal MPI group with cardiac events, 2 had abnormal coronary angiographic studies and PCI and the third had a MI on the same day as the scan. This last patient was later found to have a history of CAD and presented with $\mathrm{CP}$ in the setting of

TABLE 1

Demographics, Cardiovascular Risk Factors, and Medications ( $n=479$ )

\begin{tabular}{lc}
\hline \multicolumn{1}{c}{ Parameter } & Value \\
\hline Age $(y)$ & \\
Mean \pm SD & $44.8 \pm 11.6$ \\
Range & $20-83$ \\
Sex & \\
Male & $243(50.7)$ \\
Female & $236(49.3)$ \\
Race & \\
African-American & $290(60.0)$ \\
White & $181(37.7)$ \\
Asian/Pacific & $2(0.4)$ \\
Other & $6(1.3)$ \\
Cardiovascular risk factors & \\
Dyslipidemia (known history) & $55(11.4)$ \\
Hypertension & $187(39.1)$ \\
Diabetes & $61(12.8)$ \\
Smoking & $283(59.2)$ \\
Family history of CAD & $196(41.0)$ \\
Recent cocaine abuse & $85(17.9)$ \\
Medication & \\
$\beta$-Blockers & $11(2.2)$ \\
Calcium antagonist & $33(6.8)$ \\
Nitrate & $12(2.6)$ \\
Lipid-lowering agent & $35(7.3)$ \\
Aspirin & $17(3.5)$ \\
\end{tabular}

Values in parentheses are percentages. 
TABLE 2

Cardiac Events

\begin{tabular}{lcc}
\hline \multicolumn{1}{c}{ Cardiac event } & $\begin{array}{c}\text { Normal MPI } \\
(n=434)\end{array}$ & $\begin{array}{c}\text { Abnormal MPI } \\
(n=45)\end{array}$ \\
\hline $\mathrm{MI}$ & $1(0.3)$ & $5(11.1)$ \\
$\mathrm{PCl}$ & $2(0.6)$ & $9^{*}(20.0)$ \\
$\mathrm{CABG}$ & 0 & 0 \\
Abnormal angiogram & $0^{\dagger}$ & 0 \\
Heart failure admission & 0 & 0 \\
VT/VF arrest & 0 & 0 \\
Cardiac death & 0 & 0 \\
Total cardiac events & $3(0.7)$ & $10(22)$ \\
\multicolumn{2}{l}{.Includes MI undergoing PCl. } \\
${ }^{\dagger}$ Excludes those undergoing PCI. \\
Values in parentheses are percentages. \\
\hline
\end{tabular}

significant anemia and several comorbidities. Cardiac biomarkers were mildly elevated, and the patient was treated conservatively. The details of these 3 "false-negative" scans are shown in Table 3. CP had resolved for $>2 \mathrm{~h}$ before tracer injection in each of the 3 patients. Patients with cardiac events in the abnormal MPI group included 4 cases of MI undergoing PCI and 1 case of MI managed conservatively. The remaining 5 patients had significant coronary stenosis at catheterization and underwent PCI.

Forty-four $(9.1 \%)$ patients presented in the early morning hours and, therefore, underwent a delayed-image acquisition protocol. Details of this patient group are shown in Table 4. Most patients received tracer injection during or shortly after the cessation of CP. The average time from injection to image acquisition was $3 \mathrm{~h} 20 \mathrm{~min}$, with the longest delay remaining under $6 \mathrm{~h}$. Forty-one of these patients had normal scans and were free of cardiac events. Three had abnormal perfusion scans without cardiac events. All 3 had normal coronary angiography or normal followup stress MPI. None of the patients undergoing delayed image acquisition had a normal perfusion scan and later suffered a cardiac event.

\section{DISCUSSION}

Gated SPECT MPI has been shown to add significant diagnostic information to the standard ED CP evaluation in
TABLE 4

Delayed Image Acquisition

\begin{tabular}{lc}
\hline \multicolumn{1}{c}{ Parameter } & Value \\
\hline Resting perfusion scan results & \\
Normal & 41 \\
Abnormal & 3 \\
Cardiac events & 0 \\
Time intervals & \\
CP to injection & \\
Mean & $51 \mathrm{~min}$ \\
Injected during pain* & $64 \%$ \\
Maximum & $4 \mathrm{~h} 20 \mathrm{~min}$ \\
Injection to image acquisition & \\
Mean & $3 \mathrm{~h} 20 \mathrm{~min}$ \\
Minimum & $45 \mathrm{~min}$ \\
Maximum & $5 \mathrm{~h} 15 \mathrm{~min}$
\end{tabular}

${ }^{\star}$ Time interval $=0$.

previous studies $(3,5,6)$. One of the impediments to implementation of gated-SPECT MPI in EDs has been the logistic challenge of providing prompt image acquisition and interpretation $24 \mathrm{~h}$ per day, $7 \mathrm{~d}$ per week. The present study illustrates that-through the use of prompt tracer injection combined with flexible nuclear medicine technologist scheduling and image interpretation by local teleradiology—accurate results can be obtained. Previous reports of resting MPI in the ED excluded patients presenting in the late evening, in the early morning, or on weekends when nuclear cardiology staff are unavailable $(9,10)$. A portion of our study population underwent a delayed imaging protocol. This protocol involved radionuclide injection by trained personnel in the early morning hours with image acquisition after 6 AM when nuclear medicine technologists arrived at the hospital. ${ }^{99 \mathrm{~m}} \mathrm{Tc}$ perfusion imaging reflects relative myocardial blood flow at the time of tracer injection. There is no significant redistribution; therefore, imaging can occur some time after injection. There is a risk of image degradation with a prolonged injectionimaging interval as the agent decays. The half-life of ${ }^{99 \mathrm{~m}} \mathrm{Tc}$ is approximately $6 \mathrm{~h}$. Most of the imaging in our study, including the overnight delayed acquisition, occurred within one half-life. Of the 44 patients undergoing delayed image acquisition, 3 had abnormal scans and none of these patients

TABLE 3

Cardiac Events and Gated SPECT MPI Findings in "False-Negative Scans"

\begin{tabular}{clc}
\hline False-negative MPI events & Gated SPECT MPI findings & Timing of injection \\
\hline PCI 3 d after MPI: LCx, 70\% midvessel stenosis & Normal perfusion and function & CP resolved for $>3 \mathrm{~h}$ \\
PCI 3 d after MPI: LCx, 90\% stenosis; LAD, 20\% stenosis; & Normal perfusion and function & CP resolved for $4 \mathrm{~h}$ \\
RCA, 50\% stenosis & Normal perfusion and function & CP resolved for $5 \mathrm{~h}$
\end{tabular}

LCx = left circumflex coronary artery; LAD = left anterior descending artery; RCA = right coronary artery; NSTEMI = non-ST-elevation myocardial infarction. 
had events. More importantly, none of the patients with a normal perfusion scan had cardiac events. Our results indicate that prompt tracer injection followed by delayed image acquisition did not diminish the high negative predictive value of gated-SPECT MPI.

The overall results of the current study are consistent with previous reports. Kontos, et al. conducted a study in which low-to-moderate risk patients underwent rest MPI in the ED and were followed for $5 \mathrm{~d}$ (3). For the combined endpoint of MI or revascularization, the sensitivity and negative predictive value of rest MPI were $81 \%$ and $99 \%$, respectively - very similar to our findings. That study had a more stringent definition of positive scan findings, requiring both a perfusion defect and regional wall motion abnormality. Equivocal scans were classified as normal. The primary utility of resting cardiac perfusion imaging in a population of patients presenting to the $\mathrm{ED}$ with $\mathrm{CP}$ is in identification of patients at low risk for cardiac events. These low-risk patients may be discharged from the ED with appropriate outpatient follow-up. The current study adds to the literature a large, single-center experience showing the utility of gated SPECT MPI in identifying patients at low risk for cardiac events at 30-d follow-up.

The present study also provides details of the 3 patients who had perfusion scans interpreted as normal in the ED and later had cardiac events. Although the number of these patients is small, they are of great concern to practitioners in the ED who make decisions to release patients to home with chest discomfort. Some similarities were noted in this group. All 3 had radionuclide tracer injection after CP had resolved, in some cases several hours later. In 2 patients the cardiac event was abnormal angiography requiring PCI. One patient with a normal perfusion scan had a MI. This patient had tracer injection $5 \mathrm{~h}$ after resolution of $\mathrm{CP}$ and was admitted despite the normal MPI due to her overall clinical presentation. Furthermore, this patient was found to have a history of CAD, an exclusion criterion for this study. The appropriateness of resting MPI in this individual is questionable. Similar to our current study, previous publications have reported administration of ${ }^{99 \mathrm{~m}} \mathrm{Tc}$ tracer for several hours after the resolution of CP (2). The detailed description of the patients with normal scans having cardiac events in our study suggests that the accuracy of resting MPI could be improved further if tracer injection occurred during or shortly after CP.

This study is a single-center, case-control analysis of MPI of patients with a complaint of CP in an urban ED. Our institution handles a high volume of cardiac nuclear imaging from both the ED and the nuclear cardiology laboratory. Generalization of our findings to another practice setting may be difficult. Also, the lack of a control group and nonrandomized patient assignment fails to control for potential patient selection bias.

\section{CONCLUSION}

A normal resting gated SPECT radionuclide MPI scan predicts a very low incidence of 30-d cardiac events. Abnormal scans identify patients at higher risk for 30-d events who should receive further diagnostic testing for atherosclerotic CAD. A delayed image acquisition protocol did not diminish the accuracy of resting MPI for prediction of cardiac events in the present study. Lastly, radionuclide injection after resolution of $\mathrm{CP}$ may be associated with a higher rate of false-negative MPI results.

\section{REFERENCES}

1. Gibler WB, Runyon JP, Levy RC, et al. A rapid diagnostic and treatment center for patients with chest pain in the emergency department. Ann Emerg Med. 1995;25:1-8.

2. Pope HJ, Aufderheide TP, Ruthazer R, et al. Missed diagnoses of acute cardiac ischemia in the emergency department. $N$ Engl J Med. 2000;342:1163-1170.

3. Kontos MC, Jesse RL, Schmidt KL, Ornato JP, Tatum JL. Value of acute rest sestamibi perfusion imaging for evaluation of patients admitted to the emergency department with chest pain. J Am Coll Cardiol. 1997;30:976-982.

4. Iskander S, Iskandrian AE. Risk assessment using single-photon emission computed tomographic technetium-99m sestamibi imaging. J Am Coll Cardiol. 1998;32:57-62.

5. Mehta D, Iskandrian AE. Rest myocardial perfusion imaging in the emergency department. J Nucl Cardiol. 2004;11:224-225.

6. Kaul S, Senior R, Firschke C, et al. Incremental value of cardiac imaging in paitents presenting to the emergency department with chest pain and without STsegment elevation: a multicenter study. Am Heart J. 2004;148:129-136.

7. Kontos MC, Fratkin MJ, Jesse RL, Anderson FP, Ornato JP, Tatum JL. Sensitivity of acute rest myocardial perfusion imaging for identifying patients with myocardial infarction based on a troponin definition. J Nucl Cardiol. 2004; 11:12-19.

8. Conti A, Zanbetti M, Grifoni S, et al. Implementation of myocardial perfusion imaging in the early triage of patients with suspected acute coronary syndromes. Nucl Med Commun. 2003;24:1055-1060.

9. Udelson JE, Feldman JA, Griffith JL, et al. Myocardial perfusion imaging for evaluation and triage of patients with suspected acute cardiac ischemia: a randomized controlled trial. JAMA. 2002;288:2693-2700.

10. Knott JC, Baldey AC, Grigg LE, Cameron PA, Lichtenstein M, Better N. Impact of acute chest pain Tc-99m sestamibi myocardial perfusion imaging on clinical management. J Nucl Cardiol. 2002;9:257-262.

11. Duca MD, Giri S, Wu AHB, et al. Comparison of acute rest myocardial perfusion imaging and serum markers of myocardial injury in patients with chest pain syndromes. J Nucl Cardiol. 1999;6:570-576.

12. Hilton TC, Fulmer H, Abuan T, Thompson RC, Stowers SA. Ninety-day followup of patients in the emergency department with chest pain who undergo initial single-photon emission tomography perfusion scintigraphy with technetium 99m-labeled sestamibi. J Nucl Cardiol. 1996;3:308-311.

13. Miller TD, Christian TF, Hopfenspirger MR, Hodges DO, Hauser MF, Gibbons RJ. Prognosis in patients with spontaneous chest pain, a nondiagnostic ECG, normal cardiac enzymes, and no evidence of severe resting ischemia by quantitative technetium 99m sestamibi tomographic imaging. J Nucl Cardiol. 1998; 5:64-72. 